\title{
Vagal Responsiveness to Gavage Feeding as an Index of Preterm Status
}

\author{
JANET A. DIPIETRO AND STEPHEN W. PORGES \\ Department of Maternal and Child Health, Johns Hopkins University, Baltimore, Maryland 21205 and Institute \\ for Child Study, University of Maryland, College Park, Maryland 20742
}

\begin{abstract}
Vagal responsivity to orogastric stimulation is necessary for gastrointestinal function and may reflect autonomic regulation. Our study evaluated vagal responsivity during gavage feeding as an index of preterm clinical status. Vagal and behavioral responsivity of 26 preterm infants during gavage feeding was measured. Vagal tone was quantified with a noninvasive measure (vagal tone index) developed by Porges (Porges SW: U.S. patent 4510944,1985 ), which extracts a valid measure of cardiac vagal tone from the ECG. The study investigated whether vagal tone changed during gavage feeding and whether individual differences in the vagal tone response pattern were related to clinical outcome. Infants who demonstrated an increase in vagal tone during feeding and a decrease after feeding had significantly shorter hospitalizations. This effect was independent of weight gain trajectories. Baseline levels of vagal tone were positively associated with subsequent weight gain. Traditional risk measures did not predict length of hospitalization independently of gestational age. This study represents the first documentation that small changes in cardiac vagal tone during gavage feeding, previously imperceptible to clinicians and researchers, may be quantitatively extracted from the ECG and provide an important index of clinical status. (Pediatr Res 29: 231-236, 1991)
\end{abstract}

Abbreviations

$\hat{\mathbf{V}}$, vagal tone index

PCS, postnatal complications score

The concept of perinatal risk as a continuous dimension has developed over the last three decades (1-3). A number of perinatal events including preterm birth/low birth weight $(4,5)$, respiratory distress (6), and persistent mechanical ventilation (7) have been associated with neonatal morbidity and altered development. However, identification of risk characteristics that significantly predict morbidity in preterm infants is often confounded by large variation in outcome within risk groups (8-11).

Traditional risk scales attempt to gauge physiologic compromise, but must do so indirectly through observable events (e.g. prematurity, ventilatory assistance, etc.). These risk indicators do not directly assess the compromise of physiologic function. Rather these risk scales focus on detailing the occurrence of specific events and assume that the functional impact of each event is relatively invariant across individuals. The current study proposes that risk status can be more directly assessed by meas-

Received July 16, 1990; accepted October 19, 1990.

Reprint requests: Janet DiPietro, Johns Hopkins University, Department of Maternal \& Child Health, 624 N. Broadway, Baltimore, MD 21205.

Supported by Grant no. HD22628 from NICHHD (S.W.P.). urement of physiologic function in response to a standard clinical intervention, gavage feeding.

In the healthy, mature individual, gastric filling stimulates vagal afferents in the stomach that reflexively stimulate the vagal efferents necessary for enhanced gastrointestinal motility (12) and polypeptide release (13). Thus, in the preterm neonate, gastric filling via gavage feeding should have direct consequences on the vagus. Individual differences in the vagal response pattern might be related to clinical outcome.

Measures of short-term and long-term heart rate variability have been assumed to reflect changing vagal influences on the heart (14). Although these assumptions have a degree of physiologic validity, these measures suffer from several limitations including: 1) nonneural influences and 2) transitory state changes. A more direct and accurate approach to the study of vagal tone of the heart would be to extract a component of heart rate variability that is mediated by known vagal mechanisms.

Respiratory sinus arrhythmia is a valid and reliable window to the vagal control of the heart (15). Respiratory sinus arrhythmia is commonly observed as an increase in heart rate during inspiration due to a transitory attenuation of vagal efferent activity and a decrease in heart rate during expiration when the vagal efferent activity is expressed directly on the sinoatrial node of the heart (16). Research has demonstrated that neural blockade of the vagus via atropine, denervation, or cooling (17) greatly attenuates the amplitude of respiratory sinus arrhythmia. Given the physiologic validity of respiratory sinus arrhythmia as a measure of vagal tone, monitoring respiratory sinus arrhythmia might provide an index to changing vagal input on the gastrointestinal system during gavage feeding.

Numerous methods have been proposed to quantify respiratory sinus arrhythmia. The approach incorporated into the current research models, with time series methods, the changing heart rate trend before extracting respiratory sinus arrhythmia. This procedure, developed by Porges (18), produces an accurate estimate of vagal tone by minimizing confounding (i.e. nonneural) influences and enables the quantification of small changes over short periods of time (19). This vagal tone estimate $(\hat{V})$ is sensitive to neonatal clinical status (20), positive developmental outcome (21), and subsequent development of chronic lung disease (22). Research on vagal reactivity to circumcision in full-term infants (23) and to lumbar puncture in preterm infants (24) has demonstrated that cardiac vagal tone decreases in response to these stressful procedures, and that infants with higher baseline levels of cardiac vagal tone display greater vagal reactivity to the stressor.

The current study examines cardiac vagal tone and behavioral reactivity of the preterm infant to gavage feeding. Gavage feeding was selected because: $I$ ) insertion of the gavage tube and passage of fluid to the stomach stimulates vagal efferents (25) and 2) preterms born before approximately 34 wk gestational age are routinely gavage-fed, so administration of this procedure is not confounded with medical compromise.

In summary, we hypothesize that vagal responsivity to gavage 
feeding can provide a measurable index of risk status. Length of postpartum hospitalization is commonly used in studies that evaluate the efficacy of perinatal risk measurement $(26,27)$ and was selected as the outcome variable to reflect neonatal clinical course. Because both reactivity and recovery after response are vital components to regulation of physiologic homeostatic control (28), it was predicted that strong vagal and behavioral patterns of response and recovery to orogastric feeding in the first weeks after birth would be associated with shorter hospitalization. Specifically, a vagal surge followed by vagal suppression should characterize the response of infants with a well-integrated level of autonomic regulation. In addition, baseline levels of vagal tone were measured.

\section{MATERIALS AND METHODS}

Study design. Data were collected on 32 preterm infants. Subjects were recruited over a 6-mo period from the Neonatal Intensive Care Unit at Columbia Hospital for Women, and were preselected on the following criteria: gestational age by Dubowitz (29) <34 wk (to maximize opportunity for gavage feeding); commencement of gavage feeding within $7 \mathrm{~d}$ postpartum; appropriate weight for gestational age; and lack of congenital anomalies. These criteria eliminated infants who displayed significant perinatal asphyxia and/or required persistent mechanical ventilation. At time of testing, as part of normal clinical routine, all infants were on heart rate monitors and were being fed via gavage. The research protocol was approved by the institutional review boards of the University of Maryland and Columbia Hospital for Women. Due to poor ECG data quality or irregularity in the gavage feeding procedure, data from six subjects were incomplete. All analyses are based on the 26 subjects with complete data. The average gestational age for the sample was 30 weeks, and subjects were tested at an average of $7 \mathrm{~d}$ postpartum $(\mathrm{SD}=3.3$; mean postconceptional age $=31 \mathrm{wk} ; \mathrm{SD}=1.8)$. Perinatal characteristics are summarized in Table 1. Five subjects displayed intraventricular hemorrhage of up to grade III, but no subjects were seriously ill as demonstrated by the low incidence of ventilatory support.

Risk status was assessed by the following measures: birth weight; 1-and 5-min Apgar; a combined measure of respiratory support, which ranks subjects on a 6-point scale based on the length of time requiring oxygen, and/or ventilator support ${ }^{\prime} ;$ the Hobel Neonatal Risk Scale (26); and PCS (30). Fifty-eight \% of the subjects were female and $77 \%$ were nonwhite. The participating hospital serves a large urban population in Washington, D.C., and although a range of socioeconomic status was represented in the current sample, $42 \%$ of families received public aid.

Physiologic and state data were collected to provide information concerning initial values and reactivity to the gavage procedure. Data collection began immediately before the 1100 or $1400 \mathrm{~h}$ scheduled feeding. No medical interventions were performed during the data collection period.

After discharge, discharge data and weight gain during hospitalization data were collected from hospital records.

Physiologic recording and data quantification. Heart rate was continuously monitored as part of the routine medical care of all subjects. ECG data were recorded via the neonate's existing monitor on an FM tape recorder (Vetter model C-4, Rebersberg, PA). The ECG data were analyzed off-line on a laboratory computer that detected the peak of the $R$ wave for each cardiac cycle and timed sequential $\mathrm{R}-\mathrm{R}$ intervals (i.e. heart periods) to the nearest ms. Because vagal influences to the heart result in transitory delay in the onset of the next heart beat (i.e. longer heart period), data analyses were conducted on heart period values rather than on beat-to-beat measures of rate. Heart period

' The Respiratory Support categorical variable was coded as follows: $1=$ no $\mathrm{O}_{2}$ or mechanical ventilation except in delivery room: $2=\mathrm{O}_{2}<24 \mathrm{~h} /$ no ventilation. $3=\mathrm{O}_{2} 2-5 \mathrm{~d} /$ no ventilation: $4=$ ventilation $<24 \mathrm{~h} ; 5=$ ventilation $2-3 \mathrm{~d} ; 6=$ ventilation $>4 \mathrm{~d}$.
Table 1. Perinatal characteristics $(n=26)$

\begin{tabular}{lrrc}
\hline \multicolumn{1}{c}{ Measure } & Mean & \multicolumn{1}{c}{ SD } & Range \\
\hline Gestational age & 30.1 & 2.2 & $26-33$ \\
Birth wt (g) & 1403.4 & 306.9 & $900-1880$ \\
Apgar at 1 min & 5.6 & 2.4 & $1-9$ \\
Apgar at 5 min & 7.8 & 1.2 & $4-9$ \\
Days on O & 2.2 & 3.1 & $0-10$ \\
Days ventilated & 1.5 & 2.6 & $0-10$ \\
Hobel Risk Scale & 38.1 & 21.2 & $15-85$ \\
PCS & 2.3 & 1.7 & $0-6$ \\
Maternal age & 25.9 & 5.1 & $16-36$ \\
\hline
\end{tabular}

data represent the inverse of heart rate.

Cardiac vagal tone $(\hat{V})$ was quantified with MXedit software (Delta-Biometrics, Bethesda, MD) based on the patented procedures of Porges (18). This method consisted of the following steps: 1 ) the heart periods were converted into time-based data by sampling in successive $200-\mathrm{ms}$ intervals; 2) the trend and periodicities of heart rate slower than respiratory sinus arrhythmia were removed with a 21 -point moving polynomial; 3) these data were processed by a band-pass filter to extract the variance of heart period within the frequency band characteristic of spontaneous breathing in the neonate (i.e. $0.3-1.3 \mathrm{~Hz}$ or approximately 20 to 80 breaths/min); and 4) the natural logarithm of the band-passed variance was calculated and used as the measure of cardiac vagal tone. To increase the stability of the vagal tone estimate, cardiac vagal tone was calculated for sequential 30-s epochs and the mean of these epochs within condition was used in the statistical analyses.

Baseline physiologic data. Baseline physiologic data were collected before gavage feeding during a 10 -min period of active (rapid eye movement) sleep. Because heart period varies as a function of state, only data collected during a 10-min uninterrupted period of active sleep were used. State assessment techniques are described in the following section.

Reactive physiologic data. The gavage procedure was performed by the infant's staff nurse and involved the following: a brief period of preparation to determine tube placement; insertion of the orogastric tube; aspiration of the gastric contents to insure proper placement; passive passage of the formula; removal of the tube; and prone placement of the infant. Pacifiers were not used during the feeding. The mean duration of the feeding was $4 \mathrm{~min}$.

The continuously recorded data were parsed into three segments to allow statistical evaluation of the impact of gavage feeding on heart period and cardiac vagal tone. The $3 \mathrm{~min}$ before tube insertion, the entire gavage feeding, and the $3 \mathrm{~min}$ after tube removal provided the pregavage, gavage, and postgavage segments.

Transient cardiac decelerations to tube insertion characterized by a brief, rapid drop in heart rate were removed from calculations of the values of heart period and vagal tone for the gavage condition. A transient increase to a level of $>600$-ms interbeat (heart period) interval was used as criterion, which corresponds to approximately 100 beats $/ \mathrm{min}$. The presence of this response was recorded for each subject, but the 2-3 s of heart period data during these episodes were excluded from the mean calculations so as not to impact on the overall heart period and vagal tone values during the duration of the feeding. Given methods used to estimate cardiac vagal tone, rapid drops in heart rate with slope and period similar to breathing can greatly inflate $\hat{V}$. Therefore, this represents a conservative approach to mitigating a transient response reflective of a phasic vagal reflex independent of the more tonic response during gastric filling. Means for heart period and vagal tone within the pregavage, postgavage, and gavage period conditions were then calculated.

Behavioral data collection. Behavioral reactivity to tube insertion was independently assessed by the nurse and the observer on a 3-point scale (none, moderate, high). This score was a 
measure of intensity based on the presence of behaviors such as grimacing, motor activity, and tube pulling.

State-related behaviors were assessed every $15 \mathrm{~s}$ during data collection by one of two observers using Anderson's 12-level Behavioral State Scale (31). Observation of respiration, eye movements, and motor activity are used to categorize 12 behavioral constellations. This state scale is similar to others more commonly used with full-term infants, but was selected because it was designed for preterm infants, who often demonstrate more variable state patterns. Interrater training on state scoring took place before the study began and was maintained at 0.90 during weekly reliability checks. Predominant state during each segment was derived from the state records.

Data analysis. Heart period, vagal tone, and state during each feeding segment were analyzed using repeated measure analysis of variance to determine reactivity to the procedure. Significance of differences between each time period were tested with Neuman-Keuls multiple range tests. Correlations between perinatal measures, baseline, and reactive physiologic measures were computed. Forward multiple regression techniques were used to evaluate individual differences and to predict outcome. All statistical analyses were conducted using the SAS statistical software package (32).

\section{RESULTS}

Reactivity to gavage procedure. There were significant main effects for heart period $[F(2,25)=6.78, p<0.01]$ and cardiac vagal tone $[F(2,25)=7.58, p<0.001]$ during the recorded segments. Figure 1 presents mean values for heart period and cardiac vagal tone $(\mathrm{V})$ at each period. Significance tests between time periods indicated that the gavage segment differed from both pre- and postgavage levels of $\hat{V}$ and from pregavage heart period. Both heart period and $\hat{V}$ increased during feeding. Immediately after tube removal $\hat{V}$ decreased to below pregavage levels. The mean and SD values for the pregavage, gavage, and postgavage period were $1.1(1.0), 1.5(1.0)$, and $0.7(1.0)$ for $\hat{V}$ and $373.8(29.2), 388.6(22.1)$, and 382.7 (23.9) for heart period.

Bradycardia occurred several seconds after tube insertion in $50 \%$ of subjects. As noted above, these data were eliminated from the quantification of heart period and vagal tone in these individuals. Therefore, the significant increases in the cardiac measures are independent of these transient, but potentially biasing, influences. $t$ tests were performed to determine whether subjects who demonstrated such cardiac decelerations to tube insertion differed from those who did not. No significant group differences were detected on any perinatal variable, including gestational age, postconceptional age, and risk scales, or on either baseline levels or gavage reactivity of heart period and $\hat{V}$.

The change in heart period patterning during gavage is illustrated for a single subject in Figure 2. The heart period variability during the gavage period is apparent, as is the cardiac deceleration occurring approximately $20 \mathrm{~s}$ after tube insertion. Figure 3 details the heart period pattern for $60 \mathrm{~s}$ during the pregavage, gavage,

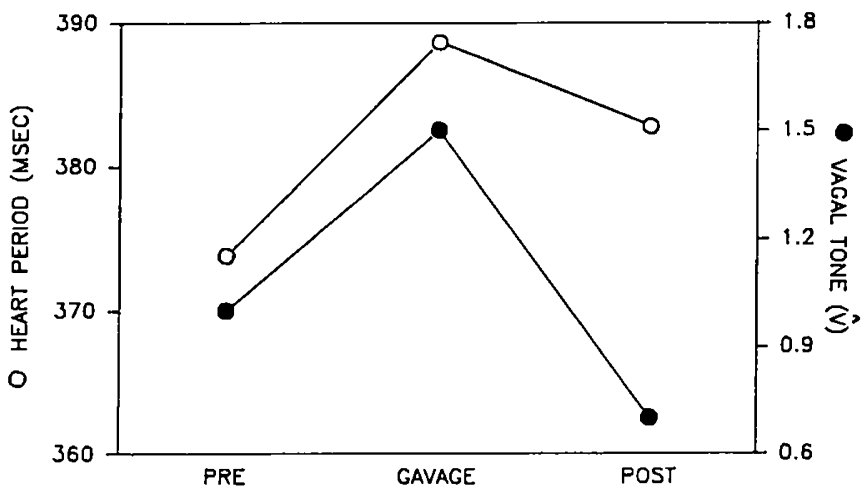

Fig. 1. Physiologic response to gavage $(n=26)$.

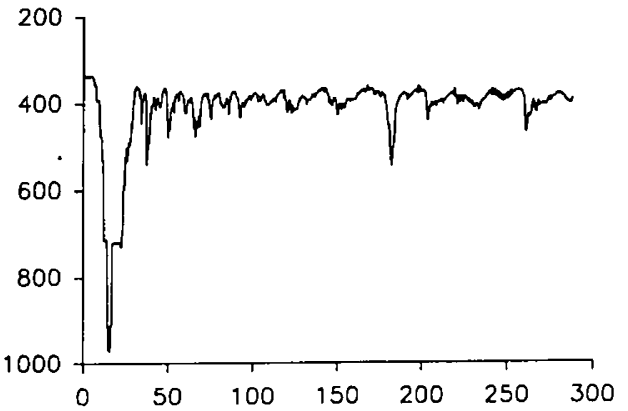

Fig. 2. Heart period pattern in response to gavage feeding.
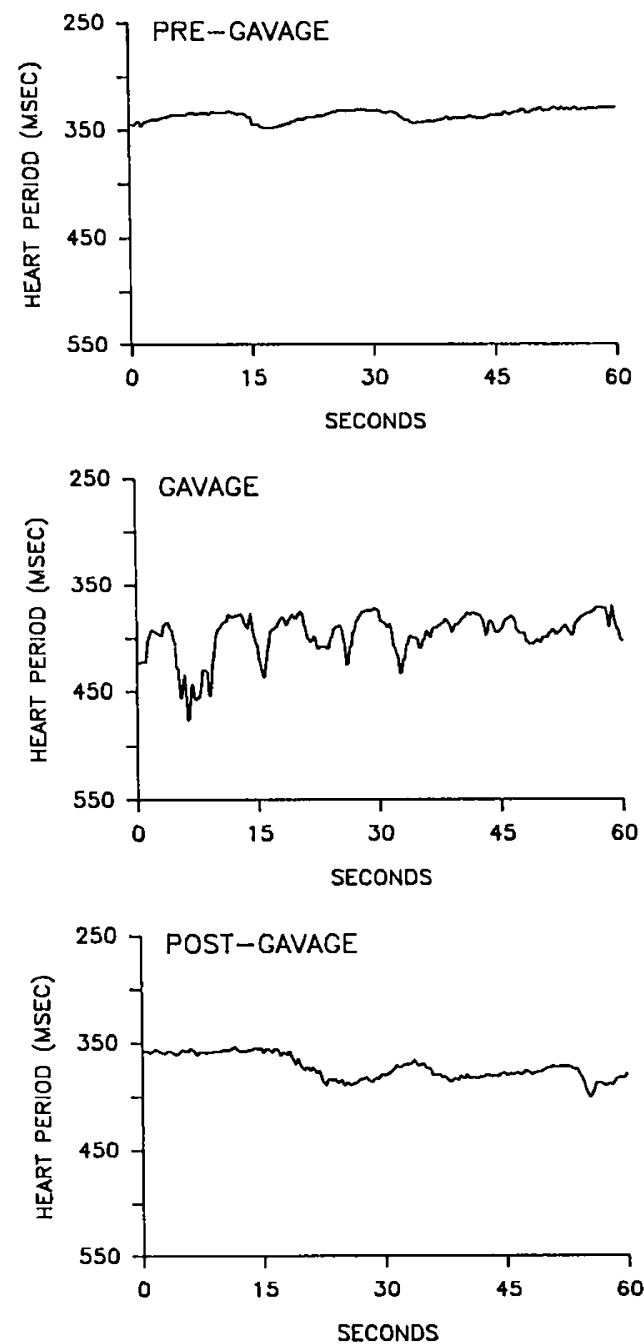

Fig. 3. Detailed illustration of $60 \mathrm{~s}$ of heart period data for each condition. Gavage data represent the 2 nd min after tube insertion.

and postgavage periods. To emphasize the effect of gavage feeding on the heart period pattern, independent of the vagal reflexes elicited by tube insertion, the 60 s illustrated during gavage were selected $1 \mathrm{~min}$ after tube insertion.

There was a trend for predominant behavioral state to change from pre- to postgavage $(p<0.06)$. As illustrated in Figure 4 , subjects tended to progress from a drowsy state during pregavage to an active (rapid eye movement) sleep state. Although heart period patterning is associated with behavioral state, the finding that the state changes proceeded linearly (i.e. from a higher to a lower state), whereas the physiologic response pattern was nonlinear, suggests that the cardiac responses to gavage were not driven simply by state changes instigated by behavioral responsiveness to being fed. 


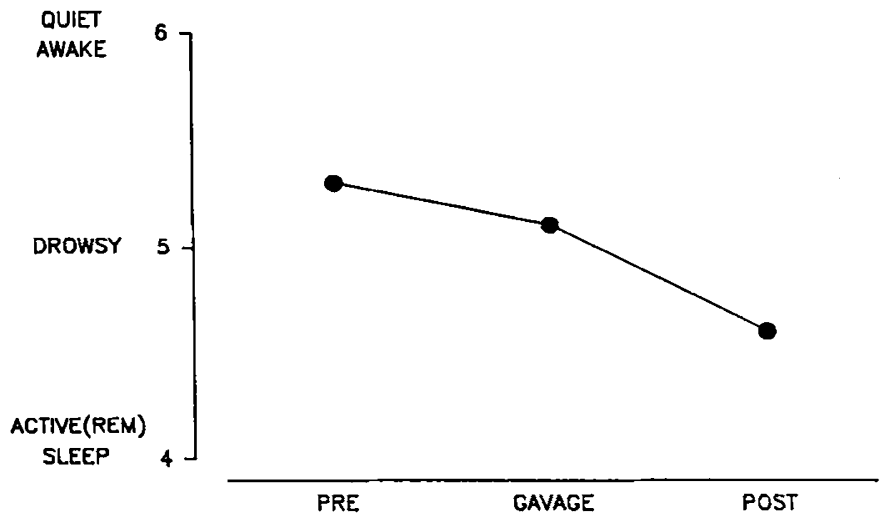

Fig. 4. State changes during gavage recording $(n=26)$.

Additional analyses based on sex, maturation, and risk level indicated no main effects or interactions for these variables on physiologic measures or predominant state. There were no group influences on heart period, $\hat{V}$, or predominant state during the three gavage segments when subjects were categorized into: $I$ ) male and female groups; 2) high- and low-risk groups (based on Hobel score and PCS; or 3) older and younger age groups (gestational age $\geq 32$ wk versus $<32$ wk or; conceptional age $\geq$ 32 wk versus $<32 \mathrm{wk}$ ). The age demarcation was set at $32 \mathrm{wk}$ because at this age there appears to be significant increases in myelination of the vagus (33) and in the cardiac vagal tone measure (20). Comparisons of behavioral reactivity between these group classifications also were not significant.

Perinatal-physiologic associations. Relationships among the perinatal and dependent measures were explored. Correlations were computed between measures of neonatal risk (gestational age, birth weight, Apgar scores, respiratory support, PCS, and Hobel Scale) and physiologic and behavioral measures. These include: baseline heart period and $\hat{\mathrm{V}}$, cardiac reactivity to gavage measures (change in heart period and $\hat{\mathrm{V}}$ from pregavage to gavage levels and from pregavage to postgavage), and the behavioral reactivity measure. The correlation matrix is presented in Table 2 , with significant relationships $(p<0.05)$ indicated.

Additional correlations, computed within the physiologic and behavioral measures, indicated the following relationships: 1) subjects who displayed greater heart period and vagal tone reactivity to tube insertion were less behaviorally responsive [heart period $(r=-0.48)$ and $\hat{V}(r=-0.42)] ; 2)$ baseline levels of heart period and $\hat{V}$ were not related to the magnitude and direction of the subseguent response to gavage; and 3 ) there was a trend for baseline $\hat{\mathrm{V}}$ to be positively related to behavioral reactivity ( $r=$ $0.34 ; p<0.10$ ).

Prediction of hospitalization. The overall response pattern of cardiac vagal tone was used to categorize subjects into three groups based on our initial hypothesis concerning vagal response and recovery. These were (A) strong responders (reactivity characterized by an increase in $\hat{\mathrm{V}}$ and recovery characterized by a decrease in $\hat{\mathrm{V}}$ to below pregavage levels, as depicted in Fig. 1) ( $n$ $=10$ ); (B) nonrebounders (initial reactivity but lack of a rebound to below pregavage levels) $(n=7)$; and (C) nonreactors (lack of vagal increase to gavage feeding) $(n=9)$.

Analysis of variance (response pattern $\times$ length of hospitalization) indicated a significant effect for vagal response pattern $[F(2,25)=3.65, p<0.05]$. Strong responders were hospitalized for significantly shorter periods of time than the other two groups. There were no differences in gestational age, conceptional age, birth weight, Apgar, Hobel Scale, PCS, or respiratory support among these groups. On the average, subjects in group A were discharged $20 \mathrm{~d}$ before subjects in groups B and C. The mean duration of hospitalization for these three groups was 28,52 , and $45 \mathrm{~d}$, respectively.

Because weight criteria are an important component to hospital discharge and represent potentially confounding factors in the significance of vagal responsivity demonstrated in the preceding analysis, weight gain trajectories from the point of testing were computed. Weight gain variables included $l$ ) weight gain/ $\mathrm{d}(\mathrm{g})$ from testing to discharge and 2) weight gain/d (g) during the week after testing. The latter variable was included to provide a standard time period in which to evaluate weight gain, inasmuch as a limitation of the former variable is assumption of a linear growth pattern.

Weight at discharge ranged from 1710 to $2365 \mathrm{~g}$ (mean $=2100$ g); mean weight gain/d was $26.2(\mathrm{SD}=6.4)$ to discharge and $17.4(\mathrm{SD}=12.2)$ in the week after testing. Analysis of variance (vagal response pattern $\times$ weight gain) indicated no relationship between vagal responsiveness and either weight gain measure $[F(2,25)=0.50,0.52$, respectively]. In contrast to vagal reactivity, baseline vagal tone was significantly correlated with both weight gain measures. Because there was a positive relationship between gestational age and baseline $\hat{\mathrm{V}}(r=0.41)$, partial correlations were computed with GA covaried. Baseline $\hat{\mathrm{V}}$ was positively associated with both discharge weight gain (partial $r=0.45 ; p<$ 0.05 ) and $7-d$ weight gain (partial $r=0.63 ; p<0.001$ ). Baseline heart period did not correlate with weight gain measures.

Although there were no differences among the vagal response patterns for either perinatal or weight gain measures, multiple regressions were conducted to insure that these factors did not systematically contribute in combination to these observed results. Vagal response pattern was entered as the final step in two forward regressions with GA, Hobel Scale, and weight gain rate (discharge weight gain used in the first equation, 7-d weight gain in the second) with length of hospitalization as the dependent measure. Vagal response pattern was entered as a two-level categorical dummy variable (i.e. full vagal response of reactivity and rebound versus incomplete response). GA was entered in step 1 as a conservative method to attribute the greatest amount of variance to it alone. In addition to GA, vagal response pattern

Table 2. Correlation matrix for perinatal risk and physiologic measures

\begin{tabular}{|c|c|c|c|c|c|c|c|}
\hline & \multirow{2}{*}{$\begin{array}{c}\text { Gestational } \\
\text { age } \\
\end{array}$} & \multirow{2}{*}{$\begin{array}{c}\text { Birth } \\
\text { wt }\end{array}$} & \multicolumn{2}{|c|}{ Apgar score } & \multirow{2}{*}{$\begin{array}{l}\text { Respiratory } \\
\text { treatment }\end{array}$} & \multirow{2}{*}{$\begin{array}{l}\text { Hobel } \\
\text { Scale }\end{array}$} & \multirow[b]{2}{*}{ PCS } \\
\hline & & & $1 \mathrm{~min}$ & $5 \mathrm{~min}$ & & & \\
\hline \multicolumn{8}{|l|}{ Baseline physiologic level } \\
\hline HP* & 0.30 & 0.14 & 0.04 & 0.13 & -0.05 & -0.06 & -0.02 \\
\hline$\hat{\mathrm{v}}$ & $0.41+$ & 0.14 & 0.12 & 0.16 & 0.01 & -0.09 & -0.02 \\
\hline \multicolumn{8}{|l|}{ Physiologic reactivity } \\
\hline HP (pregavage to gavage) & $-0.49 \ddagger$ & $-0.38 \dagger$ & -0.36 & -0.24 & 0.26 & 0.30 & 0.23 \\
\hline$\hat{\mathrm{V}}$ (pregavage to gavage) & -0.27 & -0.14 & 0.00 & 0.00 & 0.12 & 0.03 & 0.04 \\
\hline HP (pre- to postgavage) & $0.39 \dagger$ & 0.30 & 0.35 & 0.20 & -0.24 & -0.37 & -0.26 \\
\hline$\hat{\mathrm{V}}$ (pre- to postgavage) & 0.29 & 0.19 & 0.27 & 0.11 & -0.27 & $-0.47 \ddagger$ & -0.33 \\
\hline Behavioral reactivity & 0.35 & 0.20 & 0.21 & 0.13 & -0.19 & 0.05 & 0.03 \\
\hline
\end{tabular}

${ }^{*} \mathrm{HP}$, heart period.

$\dagger p<0.05$.

$\ddagger p<0.01$. 
continued to be significantly predictive of hospital discharge in each regression $(F=5.71, p<0.05)$. Neither the Hobel Risk Scale nor either weight gain trajectory contributed additional significant variance to $\mathrm{GA}$ in prediction of outcome $(F=0.90$, $1.26,0.63$, respectively).

\section{DISCUSSION}

Physiologic and behavioral reactivity to an invasive neonatal intensive care unit procedure was demonstrated in this sample of preterm infants. The typical vagal response incorporated an initial reactive component to the stressor, followed by recovery within the 3-min postgavage time period. Transient cardiac decelerations upon tube insertion were exhibited by half of the subjects, indicating that periods of vagal instability are common in this group. Because exhibition of bradycardia to the procedure was not associated with age, perinatal risk factors, or baseline physiologic levels, prediction of this phenomenon remains illusive. These episodes may represent phasic responses to mechanical stimulation resulting from tube insertion, compared with the prolonged vagal responsivity observed during gastric filling.

Although the initial surge in vagal tone and ensuing recovery demonstrates that the regulation of physiologic homeostasis exists in preterms in the perinatal period, there was variability in the degree and patterning of this response. Such variability is obscured by simple repeated measures analysis on the entire sample. As predicted, evaluation of individual differences in vagal response patterns revealed that infants who demonstrated vagal responsiveness characterized by a vagal increase to feeding followed by vagal rebound to below initial levels had a more optimal clinical course than those who displayed either no initial reactivity or no rebound. Specifically, these subjects were discharged an average of $20 \mathrm{~d}$ earlier than subjects who did not demonstrate the complete vagal response. These results are particularly striking because the sample consisted of relatively healthy preterm infants, and the infants in the first group were neither heavier, older, nor less medically compromised at birth, nor did they demonstrate a different pattern of weight gain during hospitalization than those in the other two groups.

Maturity at birth is clearly the most important determinant of hospitalization duration, yet these results suggest that the ability of the nervous system to maintain homeostatic function via negative feedback is independently associated with short-term clinical outcome. Standard risk factors and scales were ineffective in predicting either behavioral or physiologic reactivity to the gavage procedure. However, the question remains whether measurement of vagal reactivity represents a window into the integrity of the nervous system, or whether vagal reactivity exerts an indirect role on hospitalization by impacting digestive function. That is, was the observed relationship a function of enhanced weight gain, or are clinical judgments of medical stability that precipitate hospital discharge based on underlying regulation of the nervous system? Results of this study suggest the latter; a strong vagal response to feeding should be interpreted as independent of digestive function (as monitored by weight gain) and represents neural regulation to a perturbation (in this case, the disruption is gavage feeding) of the physiologic system. The role of homeostatic function in these findings is further supported by the negative relationship observed between vagal recovery after the stressor and higher Hobel Risk Score. Conversely, baseline (e.g. undisturbed) levels of vagal tone were positively correlated with weight gain and may represent overall mediation of digestive function for the infant. This relationship may reflect the input of the vagus in stimulating the secretion of gastrointestinal hormones (13).

This pattern of results suggests that measurement of physiologic reactivity provides information unique from measures of baseline physiologic level. In addition, although baseline cardiac vagal tone was positively correlated with gestational age $(r=$ 0.41 ), vagal reactivity was not associated with gestational age or any other perinatal risk factor. Moreover, the prediction of hospitalization was based on vagal responsiveness, not baseline level. Thus, vagal tone reactivity in response to clearly defined external stimulation provides an index of the dynamic selfregulatory properties of the infant's autonomic nervous system. This property appears to be independent of endogenous regulation, which may be better characterized by baseline levels of cardiac vagal tone.

In summary, our study represents the first documentation that small changes in cardiac vagal tone during orogastric stimulation, previously imperceptible to clinicians and researchers, may be quantitatively extracted from the ECG and can provide an index of clinical status. The results demonstrate that vagal reactivity to gavage feeding is related to length of hospitalization. This effect was not determined by individual differences in gestational age, behavioral reactivity, state, or other measures of risk. Multiple regressions indicate that in conjunction with gestational age, vagal response patterning is a better predictor of clinical course than either traditional risk scales or weight gain trajectories. Thus, physiologic regulation, evidenced by a vagal surge followed by vagal suppression, may provide a marker for individual differences in neural integrity even for preterm infants of similar gestational age. These findings suggest that efforts at predicting neonatal morbidity should be directed at quantifying actual compromise, rather than assigning risk based on factors associated with broad-based group differences. This approach has implications for clinical decision-making concerning infant status and for allocation of resources during neonatal care. We are suggesting that before efforts to predict the complicated realm of developmental outcome can be successful, current methods of designating risk in the perinatal period must be carefully reevaluated, and more documentation of the relationship between perinatal events and short-term outcome is essential.

Acknowledgment. The authors thank Dr. John Scanlon, Columbia Hospital for Women, Washington, D.C. for his support.

\section{REFERENCES}

1. Lilienfeld AM, Parkhurst E 1951 A study of the association of factors of pregnancy and partition with the development of cerebral palsy: a preliminary report. Am J Hyg 53:262-282

2. Pasamanick B, Knobloch H 1961 Epidemiologic studies on the complications of pregnancy and the birth process. In: Caplan $G$ (ed) Prevention of Mental Disorders in Children. Basic Books, New York

3. Sameroff AJ, Chandler MJ 1975 Reproductive risk and the continuum of caretaking casualty. Rev Child Dev Res 4:187-244

4. Philip AGS, Little GA, Polivy DR, Lucey JF 1981 Neonatal mortality risk for the eighties: the importance of birth weight/gestational age groups. Pediatrics 68:122-130

5. McCormick MC 1985 The contribution of low birth weight to infant mortality and childhood morbidity. N Engl J Med 312:82-90

6. Field T, Hallock N, Ting G, Dempsey J, Dabiri C, Shuman HH 1978 A firstyear follow-up of high-risk infants: formulating a cumulative risk index. Child Dev 49:119-131

7. Bozynski ME, Nelson MN, Matalon TA, O’Donnell KJ, Naughton PM, Vassan U, Meier WA, Ploughman L 1987 Prolonged mechanical ventilation and intracranial hemorrhage: impact on developmental progress through 18 months in infants weighing 1,200 grams or less at birth. Pediatrics 79:670676

8. Hack M, Fanaroff AA, Merkatz IR 1979 The low-birth-weight infant-evolution of a changing outlook. N Engl J Med 301:1162-1166

9. Noble-Jamieson CM, Lukeman D, Silverman M, Davies PA 1982 Low birth weight children at school age: neurological, psychological, and pulmonary function. Semin Perinatol 6:266-273

10. Marlow N, D'Souza SW, Chiswick ML 1987 Neurodevelopmental outcome in babies weighing less than $2001 \mathrm{~g}$ at birth. Br Med J [Clin Res] 294:15821586

11. Kopp CB, Parmelee AH 1979 Prenatal and perinatal influences on behavior. In: Osofsky J (ed) Handbook of Infant Development. Wiley, New York

12. Roman C, Gonella J 1987 Extrinsic control of digestive tract motility. In: Johnson LR, Christensen J, Jacobsen ED, Schultz SG (eds) Physiology of the Gastrointestinal Tract. Raven Press, New York, pp 507-553

13. Uvnas-Moberg K 1989 Gastrointestinal hormones in mother and infant. Acta Pediatr Scand [Suppl] 351:88-93

14. Cabal LA 1987 Neonatal clinical cardiopulmonary monitoring. In: Fanaroff AA, Martin RJ (eds) Neonatal-Perinatal Medicine. CV Mosby, St. Louis, pp 343-359 
15. Porges SW 1986 Respiratory sinus arrhythmia: physiological basis, quantitative methods, and clinical implications. In: Grossman P, Janssen K, Vaitl D (eds) Cardiorespiratory and Cardiosomatic Psychophysiology. Plenum, New York

16. Katona PG, Poitras JW, Barnett GO, Terry BS 1970 Cardiac vagal efferent activity and heart period in the carotid sinus reflex. AM J Physiol 218:10301037

17. Katona PG, Jih F 1975 Respiratory sinus arrhythmia: noninvasive measures of parasympathetic cardiac control. J Appl Psychol 39:301-305

18. Porges SW 1985 Method and apparatus for evaluating thythmic oscillations in a periodic physiological response systems. U. S. patent 4510944

19. Porges SW, Bohrer RE 1990 Analyses of periodic processes in psychophysiological research. In: Cacioppo JT, Tassinary LG (eds) Principles of Psychophysiology: Physical, Social, and Inferential Elements. Cambridge University Press, New York

20. Porges SW 1983 Heart rate patterns in neonates: a potential diagnostic window to the brain. In: Field T, Sostek A (eds) Infants Born at Risk: Physiological, Perceptual, and Cognitive Processes. Grune \& Stratton, New York, pp 3-22

21. Fox NA, Porges SW 1985 The relation between neonatal heart period patterns and developmental outcome. Child Devel 56:28-37

22. Porter F, Ultmann M, Miller JP, Arfken C, Cohlan B, Altman D 1989 Vagal tone in preterm infants at risk for chronic lung disease. Pediatr Res 25:227A(abstr)
23. Porter FL, Porges SW, Marshall RE 1988 Newborn pain cries and vagal tone: parallel changes in response to circumcision. Child Devel 59:495-505

24. Porter FL, Porges SW 1988 Neonatal cardiac responses to lumbar puncture. Infant Behav Dev 11 :(special ICIS issue) 261

25. Hay WW, Bonnabel C 1985 Neonatal nutrition. In: Merenstein GB, Garnder SL (eds) Handbook of Neonatal Intensive Care. CV Mosby Co, Princeton, pp 127-162

26. Hobel CJ, Hyvarinen MA, Okada DM, Oh W 1973 Prenatal and intrapartum high-risk screening. Am J Obstet Gynecol 117:1-9

27. Strobino DM, Baruffi G 1984 Evaluation of a measure of neonatal morbidity. Med Care 22:818-826

28. Machin KE 1964 Feedback theory and its application to biological systems. Homeostasis and feedback mechanisms. Symp Soc Exp Biol 18:421-446

29. Dubowitz IMS, Dubowitz V, Goldberg CG 1970 Clinical assessment of gestational age in the newborn infant. J Pediatr 77:1-10

30. Littman B, Parmelee AH 1978 Medical correlates of infant development. Pediatrics 61:470-474

31. Gill NE, Behnke M, Conlon M, McNeely JB, Anderson GC 1988 Effect on nonnutritive sucking on behavioral state in preterm infants before feeding Nurs Res 37:342-350

32. PC SAS System Users Guide 1986. SAS Institute, Cary, NC

33. Sachis PN, Armstrong DL, Becker LE 1982 Myelination of the human vagus nerve from 24 weeks post-conceptional age to adolescence. J Neuropath Exp Neurol 41:466-472 